\title{
Disparity of anemia prevalence and associated factors among rural to urban migrant and the local children under two years old: a population based cross-sectional study in Pinghu, China
}

Shiyun $\mathrm{Hu}^{1}$, Hui Tan ${ }^{1,3}$, Aiping Peng ${ }^{1,4}$, Hong Jiang ${ }^{1,3}$, Jianmei Wu ${ }^{5}$, Sufang Guo ${ }^{6}$ and Xu Qian ${ }^{1,2^{*}}$

\begin{abstract}
Background: Number of internal rural to urban migrant children in China increased rapidly. The disparity of anemia prevalence among them and children of local permanent residents has been reported, both in big and middle-size cities. There has been no population-based study to explore the associated factors on feeding behaviors in small size cities of China. This study aimed to identify whether there was a difference in the prevalence of anemia between children of rural to urban migrant families and local children under 2 years old in a small coastal city in China, and to identify the associated factors of any observed difference.

Methods: A community-based, cross-sectional survey was conducted in Pinghu, a newly-developing city in Zhejiang Province, China, among the caregivers of 988 children (667 who were identified as children of migrants and 321 locals) aged 6-23 months. Disparity of anemia prevalence were reported. Association between anemia prevalence and socio-economic status and feeding behaviors were explored among two groups respectively.

Results: Anemia prevalence among the migrant and local children was $36.6 \%$ and $18.7 \%$ respectively (aPR 1.86, 95\% Cl 1.40 to 2.47). Results from adjusted Poisson models revealed: having elder sibling/s were found as an associated factor of anemia with the aPR $1.47(95 \% \mathrm{Cl} 1.16$ to 1.87 ) among migrant children and 2.58 (95\% Cl 1.37 to 4.58 ) among local ones; anemia status was associated with continued breastfeeding at 6 months (aPR $=1.57,95 \% \mathrm{Cl} 1.15$ to 2.14 ) and lack of iron-rich and/or iron-fortified foods ( $\mathrm{aPR}=0.68,95 \% \mathrm{Cl} 0.50$ to 0.89 ) among the migrant children but not among local ones.

Conclusion: Anemia was more prevalent among migrant children, especially those aged 6-11 months. Dislike their local counterparts, migrant children were more vulnerable at early life and seemed sensitive to feeding behaviors, such as, over reliance on breastfeeding for nutrition after aged 6 months, lack of iron-rich and/or iron-fortified foods. Future strategies to narrow the gap of anemia prevalence between the migrant and local children should target more susceptible groups and through improvement of feeding practices among younger children in those kinds of newly-developing areas of China.
\end{abstract}

\section{Background}

Internal and cross-border migration is closely associated with globalization, economic development, urbanization and social-economic disparities. Along with market reform, China has witnessed rapid unbalanced economic growth, not only between urban and rural areas, but also

\footnotetext{
* Correspondence: xqian@fudan.edu.cn

${ }^{1}$ School of Public Health, Fudan University, Shanghai, China

${ }^{2}$ Global Health Institute, Fudan University, Shanghai, China

Full list of author information is available at the end of the article
}

between different regions. The emerging private markets for housing and employment have opened new opportunities for social mobility in China since the 1980s [1]. Millions of rural residents have migrated to urban areas, primarily from the less developed central-western parts to the well-developed eastern coastal region seeking employments opportunities $[2,3]$. The number of internal rural to urban migrants has increased dramatically in China within last few decades, reaching 221 million (17\% of total population in 2011) [4]. This number is anticipated to double 
within the next 10 years [5] and with a greater impact on small-size cities [6]. Increasingly, migrants move with their family members to the cities in which they work, leading to an increasing number of migrant children residing in those cities [7]. For example, in Zhejiang Province, which holds the second largest internal migrants population in China, there were more than 1.2 million migrant children under 14 years old in 2010, 5 times more than that in 2000 [8].

The process of migration has a mixed influence in children's health. It may provide opportunities for better access to health care services and a more diversified diet [9]. On the other hand, social factors such as the fundamental change in living environment and a lack of family support may result in poorer health due to changes in feeding practices $[10,11]$. The unique "Hukou" (household registration) system in China also complicates the situation. The Hukou determines the social welfare packages such as healthcare, education, employment, and housing, and differs for agricultural or non-agricultural residences [12], which causes difference on social benefits when rural residents with an agricultural Hukou move to the cities [13]. Migrant children, therefore, share very low utilization of healthcare in the immigrant cities [14].

Anemia has a high prevalence among young children specifically in Africa and Asia. World Health Organization (WHO) has estimated that, globally, 293 million preschool aged children are anemic and 16.8 million of them live in China. More than $40 \%$ of the world's anemia burden is caused by iron deficiency. Anemia in young children may have a long-term effect on their neurological development and behavior, some of which is irreversible [15]. The disparity of anemia prevalence among children of migrant workers (migrant children) and children of local permanent residents (local children) has been reported, both in big $[16,17]$ and middle-size cities $[18,19]$. However, there has been no population-based study to explore the associated factors especially on feeding behaviors among young migrant and local children in small size cities of China. In 2011, a project financed by UNICEF was launched to improve the health of migrant children. Based on this project, we reported the results of a survey conducted in a small coastal city in China. This survey aimed to generate evidence for future policy formulation on anemia prevention, by measuring the anemia of migrant and local children aged 6-23 months, and identify associated factors focusing on social-economic status and feeding behaviors.

\section{Methods}

\section{Study site}

This study was conducted in Pinghu, a coastal city in eastern Zhejiang Province, which has a high density of immigrants [20]. 303,000 migrants account for almost $40 \%$ of total residents, with a higher proportion of women and children (more than $60 \%$ of the city's children and half of the women of childbearing age) in 2011.

\section{Study population and sampling}

Our study population comprised children aged 6-23 months and their main caregivers. Children were selected with the following inclusion criteria: migrant children where: neither parents' Hukou was in Pinghu and at least one parent living in Pinghu for more than 6 months; and local children where: at least one parent was registered as a Pinghu permanent resident. Children were excluded if they were seriously ill or hospitalized or away from the city. If there were two or more children in one family, only the youngest was included. Age of 6 to 23 months was applied because children in this age range are at highest risk of anemia [15].

All 193 suburbs within 10 communities (still named town or township) served as clusters. In each Resident Administrative Committee (the smallest government administration agency) the list of eligible children within their areas was compiled. Information of local children was accessed from the computerized residence registration system and the migrant children mainly from the latest immunization programme and then double-checked with the records from migration administration agencies, family planning management committees and county child health service station.

Multi-stage sampling method was conducted based on the $\mathrm{WHO}$ advocated cluster sampling design. The number of samples required per cluster was calculated according to the 2009 WHO Maternal, Newborn and Child Health Household Survey Guidelines [21]. Sampling frames were generated for the migrant and the local groups separately. As a survey with multiple indicators, we assumed the proportion of children with stunting for migrant children to be $15 \%$ and local children to be $7 \%$, and prevalence of anemia for migrant children to be $20 \%$ and local children to be $10 \%$ [22-25]. The study anticipated a design effect of two, and a 90\% response rate. Therefore, 25 suburbs were sampled for the migrant children and 25 for the local children, based on probability proportional to size (PPS). Within each selected suburbs, 48 migrant and 24 local children were selected separately by revised random walking method, where the sampling start point was randomly selected from the registered addresses.

\section{Data collection and quality control}

The fieldwork was conducted in local community health centers (CHCs) between October and November, 2011, with a pilot study conducted in 25 households from August 2 to 10, 2011. All participants were interviewed face to face at their nearest $\mathrm{CHCs}$, and the hemoglobin of each child was measured after informed consent. Interviewers (physicians from local $\mathrm{MCH}$ institutes and 
$\mathrm{CHCs}$ ) and quality controllers (lecturers and senior postgraduate students from School of Public Health, Fudan University) were trained prior to conducting the survey. The survey questionnaires were routinely checked on the field and audited by external quality controllers. Reinterviews either by phone or home visits were conducted if transcription or logical problems were identified in the surveys, or where missing values were found during data cleaning or data analysis stage.

\section{Measurement}

\section{Dependent variables}

Hemoglobin $(\mathrm{Hb})$ concentration of children was measured by photometer (capillary Hb level (HemoCue ${ }^{\oplus} \mathrm{Hb} 201+$ [HemoCue, Angelholm, Sweden], precision of $1 \mathrm{~g} / \mathrm{L}$ ). About $10 \mu / \mathrm{l}$ blood sample of each child was collected from the left ring or middle finger by HemoCue Microcuvettes. Since Pinghu is at sea level, altitude adjustment was skipped. Children with hemoglobin level $<110 \mathrm{~g} / \mathrm{L}$ (WHO recommendation [26]) were defined as anemia.

\section{Independent variables}

A structured questionnaire was designed for primary caregivers, who were mostly the mothers. The questionnaire consisted of socio-demographic information (children's date of birth and gender), background of mother (education, occupation, type of Hukou) and family (annual income and family structure), child health care received and practices on feeding (including breastfeeding).

Age of children was coded into trisections (' 6 months ', '12 months $\sim$ ' and '18 23 months'). Education of mother was recoded into either 'low' (illiteracy, primary school); 'medium' (junior middle school); or, 'high' (senior middle school or higher), based on five categories of education levels in the questionnaire. Occupation of mother was recoded into "employed" or "not work for payment" based on a question with five occupational categories. One household whose members cooked and eat together was defined as one family. Annual family income was collected and categorized by P25 (RMB 50,000 Yuan) and P75 (RMB 100,000 Yuan) (percentile) of annual family income of local permanent residents $(<\mathrm{P} 25$ as poor, P25 to P75 as middle and > P75 as rich). Number of family member and relatives' appellation were collected and coded into two indicators. Family structure was recoded into parents only or living not only with parents. This indicates the caregivers of children. Number of children was recoded into one-child family and family had more than one child that indicates number of children that the family needs to look after.

Child health care (growth and development monitoring and nutrition counseling) received each year after birth was collected. Timely child health care was defined in accordance with China National Child Health Care Protocol version 2011 ( $>3$ times by aged $6-7$ months; $>4$ times by aged 8-11 months; $>5$ times by aged $12-17$ months; $>6$ times by aged $18-23$ months).

Details on feeding practices were collected based on a 24-hour dietary recall (WHO recommendation: the initiation of breastfeeding module and infant and young child feeding (IYCF) module) [27]. Four binominal variables for feeding practices including breastfeeding at 6 months, consumption of iron-rich and/or iron-fortified foods, minimum dietary diversity (MDD) and minimum meal frequency (MMF) were calculated as recommended by WHO [27]. Breastfeeding at 6 months: for children aged 6-23 months continually fed breast milk after aged 6 months. Consumption of iron-rich and/or iron-fortified foods means proportion of children aged 6-23 months who receive an iron-rich food or iron-fortified foods that is specially designed for infants and young children, or that is fortified in the home. Iron-rich foods was define as flesh foods, including any organ meats such as liver, kidney, heart, or others; any meat, such as beef, pork, lamb, goat, chicken, or duck; fresh or dried fish, shellfish or seafood. Iron-fortified foods include commercially fortified foods specially designed for infants and young children which contain iron, or foods fortified in the home with a micronutrient powder containing iron or a lipid-based nutrient supplement containing iron. MDD defined as proportion of children aged 6-23 months who receive foods from 4 or more food groups. (Among a total of 7 food groups: dairy products, legumes and nuts, flesh foods, eggs, vitamin A rich fruits and vegetables, cereals and tubers, and other fruits and vegetables). MMF is proportion of breastfed and non-breastfed children 6-23 months of age who receive solid, semi-solid, or soft foods (but also including milk feeds for non-breastfed children) the minimum number of times or more. For breastfed children, the minimum number of times varies with age (two times if aged 6-8 months and three times if aged 9-23 months). For non-breastfed children, the minimum number of times does not vary by age (four times for all children aged 6-23 months).

\section{Statistical analysis}

Table 1 summarizes the definition and coding of variable used. Descriptive analysis was conducted to present anemia prevalence among young migrant and local children through Chi square test. Poisson regression and Cochran's Mantel-Haenszel (CMH) test were used to compare the anemia prevalence between migrant and local children aged 6-23 months. Crude Prevalence Ratios between potential risk factors and outcomes were first calculated by using Poisson regression. Adjusted Poisson regression models were then developed to assess the association between social-economic status, feeding behaviors and anemia. Either significant variables (Crude Prevalence 
Table 1 Definition of variables used in the analysis

\begin{tabular}{|c|c|c|c|}
\hline \multirow{2}{*}{$\begin{array}{l}\text { Variable } \\
\text { Dependent variables }\end{array}$} & \multicolumn{2}{|l|}{ Values } & \\
\hline & & & \\
\hline Anemia (hemoglobin level <110 g/L) & 1. Yes & 0. No & \\
\hline \multicolumn{4}{|l|}{ Independent variables } \\
\hline Age of child (months) & 1. $6 \sim 11$ & 2. $12 \sim 17$ & 3. $18 \sim 23$ \\
\hline Gender of child & 1. Boy & 0. Girl & \\
\hline Education of mother & 1. Low & 2. Medium & 3. High \\
\hline Occupation of mother & 1. Not work for payment & 0. Employed & \\
\hline Annual family income (thousand RMB Yuan) & 1. Poor $<50$ & 2. Middle $50-100$ & 3. Rich $>100$ \\
\hline Caregivers & 1. Parents only & 0. Parents \& others & \\
\hline Elder sibling $/ s^{a}$ & 1. Yes & 0. No & \\
\hline Timely child health care ${ }^{b}$ & 1. Yes & 0. No & \\
\hline Breastfeeding at 6 months $^{c}$ & 1. Yes & 0. No & \\
\hline Iron $^{d}$ & 1. Yes & 0. No & \\
\hline $\mathrm{MDD}^{\mathrm{e}}$ & 1. Yes & 0. No & \\
\hline$M_{M F^{f}}$ & 1. Yes & 0. No & \\
\hline
\end{tabular}

${ }^{\mathrm{a}}$ Elder sibling/s: family had more than one child indicate the investigated child at least has one elder sibling/s.

${ }^{\mathrm{b}}$ Timely child health care: child health care received by age(month) in time.

'Breastfeeding at 6 months: for children aged 6-23 months fed breast milk at aged 6 months.

dIron: consumption of iron-rich and/or iron-fortified foods.

${ }^{\mathrm{MDD}}$ : minimum dietary diversity.

${ }^{\mathrm{f}} \mathrm{MMF}$ : minimum meal frequency.

Ratios $>1$ ) or variables were identified as risk factors of childhood anemia in the literature [28-30] were retained. Three multiple Poisson regression models were developed and performed respectively among migrant and local children. Model 1 was used to explore the association between anemia and social-economic status including age and sex of children; education level and occupation of mother; annual family income, family structure (caregiver/s) and number of children (with or without sibling/s). For the feeding behaviors, breastfeeding at 6 months and iron intake could possibly be the proxy of the two other feeding indicators. Therefore two models developed respectively to assess the association between breastfeeding at 6 months and iron intake (model 2), or MDD and MMF (model 3) with anemia. Prevalence Ratios (PR) was calculated adjusted for timely child health care and all factors in model 1. No interactions between the breastfeeding at 6 months and iron intake or MDD and MMF in both groups were found. Due to ongoing economic development and population mobilization, mother's Hukou (agricultural and nonagricultural) couldn't distinctly reflect the differences, such as urban/rural dwelling and economic status, of two types of people. As we have adjusted for education and job status as well as feeding practices, we decide not to analyse the association of the outcome with type of mother's Hukou. Crude Prevalence Ratios (cPR) and adjusted Prevalence Ratios (aPR) with 95\% CI were reported. STATA for windows version 10.1 (Stata Corporation, College Station) was used for statistical analysis.

\section{Ethics considerations}

The study protocol and informed consent procedures were approved by the Ethics Committee (IRB), School of Public Health, Fudan University. Informed consent was acquired from each main caregiver of the children.

\section{Results}

\section{General characteristics}

A total of 988 children (667 migrant and 321 local) and their caregivers were investigated. Characteristics of the study population are presented in Table 2 . There were distinct socio-demographic differences between the two populations (migrant and local). The age distribution of the two groups was consistent with the sample frame. There were more young children in the migrant group while the local children were evenly distributed in each age group. Both groups had slightly more boys; however, no statistic significance was found.

Migrant mothers had low education comparing with local mothers $(26.2 \%$ vs. $4.4 \%)$. Less migrant mothers were employed compared with the local mothers and consequently family income level was significantly lower in migrant families. More migrant children (55.8\%) lived only with parents, versus $9.0 \%$ for the local $(\mathrm{P}<0.001)$. More migrant children had elder siblings than local 
Table 2 Characteristics and related practices of study population $(\mathrm{N}=988)$

\begin{tabular}{|c|c|c|c|c|c|}
\hline & & $\begin{array}{c}\text { Total } \\
\%(\mathrm{~N}=988)\end{array}$ & $\begin{array}{c}\text { Migrant } \\
\%(n=667)\end{array}$ & $\begin{array}{l}\text { Permanent } \\
\%(n=321)\end{array}$ & $P^{*}$ \\
\hline \multicolumn{6}{|l|}{ Children } \\
\hline Age & $6 \sim$ & 41.4 (409) & 44.7 (298) & $34.6(111)$ & 0.009 \\
\hline \multirow[t]{2}{*}{ (Months) } & $12 \sim$ & $30.4(300)$ & $29.1(194)$ & $33.0(106)$ & \\
\hline & $18 \sim$ & $28.2(279)$ & $26.2(175)$ & $32.4(104)$ & \\
\hline \multirow[t]{2}{*}{ Gender } & Boy & $55.6(549)$ & $56.2(375)$ & $54.2(174)$ & 0.550 \\
\hline & Girl & 44.4 (439) & $43.8(292)$ & $45.8(147)$ & \\
\hline \multicolumn{6}{|l|}{ Mother } \\
\hline \multirow[t]{3}{*}{ Education } & Low & $19.1(189)$ & $26.2(175)$ & $4.4(14)$ & $<0.001$ \\
\hline & Medium & $55.9(552)$ & $62.9(419)$ & $41.4(133)$ & \\
\hline & High & $25.0(247)$ & 10.9 ( 73) & $54.2(174)$ & \\
\hline \multirow[t]{2}{*}{ Occupation } & Employed & $55.6(549)$ & $45.0(300)$ & 77.6 (249) & $<0.001$ \\
\hline & Not work for payment & 44.4 (439) & $55.0(367)$ & $22.4(72)$ & \\
\hline \multicolumn{6}{|l|}{ Family } \\
\hline \multirow[t]{3}{*}{ Annual income } & Poor & $48.1(475)$ & $59.8(399)$ & 23.7 ( 76) & $<0.001$ \\
\hline & Middle & $34.5(341)$ & $28.5(190)$ & $47.0(151)$ & \\
\hline & Rich & $17.4(172)$ & $11.7(78)$ & $29.3(94)$ & \\
\hline \multirow[t]{2}{*}{ Caregivers } & Parents only & $40.6(401)$ & $55.8(372)$ & $9.0(29)$ & $<0.001$ \\
\hline & Parents \& others & $59.4(587)$ & $44.2(295)$ & $91.0(292)$ & \\
\hline \multirow[t]{2}{*}{ Elder sibling/s } & Yes & $30.2(298)$ & $37.2(248)$ & $15.6(50)$ & $<0.001$ \\
\hline & No & $69.8(690)$ & $62.8(419)$ & $84.4(271)$ & \\
\hline \multicolumn{6}{|c|}{ Child health care $\&$ feeding } \\
\hline \multirow[t]{2}{*}{ Timely child health care } & Yes & $11.0(109)$ & $5.2(35)$ & $23.1(74)$ & $<0.001$ \\
\hline & No & $89.0(879)$ & $94.8(632)$ & $84.4(247)$ & \\
\hline \multirow[t]{2}{*}{ Breastfeeding at 6 months } & Yes & $74.4(735)$ & $78.4(523)$ & $66.0(212)$ & $<0.001$ \\
\hline & No & $25.6(253)$ & $21.6(144)$ & 34.0 (109) & \\
\hline \multirow[t]{2}{*}{ Iron } & Yes & $88.0(869)$ & $83.5(557)$ & $97.2(312)$ & $<0.001$ \\
\hline & No & $12.0(119)$ & $16.5(110)$ & $2.8(9)$ & \\
\hline \multirow[t]{2}{*}{ MDD } & Yes & $77.3(764)$ & $72.1(481)$ & $88.2(283)$ & $<0.001$ \\
\hline & No & $22.7(224)$ & $27.9(186)$ & 11.8 ( 38$)$ & \\
\hline \multirow[t]{2}{*}{ MMF } & Yes & $77.8(769)$ & $73.5(490)$ & 86.9 (279) & $<0.001$ \\
\hline & No & $22.2(219)$ & 26.5 (177) & $13.1(42)$ & \\
\hline
\end{tabular}

"Pearson Chi-square test.

children (37.2\% vs. $15.6 \%, \mathrm{P}<0.001)$. Less migrant children had received timely growth monitor and nutrition counseling, much lower than the local children $(5.2 \%$ vs. $23.1 \%, \mathrm{P}<0.001)$. Compared to their local counterparts, more migrant children were breastfeed at 6 months $(78.4 \%$ vs. $66.0 \%, \mathrm{P}<0.001)$. For other feeding practices, less migrant children were provided with iron-rich and/or iron-fortified foods (83.5\% vs. 97.2\%, $\mathrm{P}<0.001$ ), or had achieved minimum dietary diversity (72.1\% vs. $88.2 \%, \mathrm{P}<0.001)$ and minimum meal frequency $(73.5 \%$ vs. $86.9 \%, \mathrm{P}<0.001)$ on the previous day of the survey.

\section{Prevalence of anemia}

Table 3 revealed that 244 (36.6\%) migrant children and $60(18.7 \%)$ of the local children were diagnosed as anemic. Adjusted for age and gender of children, migrant children were found to be 1.86 times more likely to have anemia compared with their local counterparts (95\% CI 1.40 to 2.47). This disparity remained within almost each age group at statistically significant levels. Within both migrant and local groups younger children were more likely to have anemia. At the same time, younger migrant children had higher risk of anemia prevalence compared with local children and the risks decline as children getting 
Table 3 Comparison of anemia status between migrant and local children aged 6-23 months*

\begin{tabular}{|c|c|c|c|c|c|c|}
\hline \multirow{2}{*}{$\begin{array}{c}\text { Age } \\
\text { (month) }\end{array}$} & \multicolumn{2}{|c|}{ Migrant } & \multicolumn{2}{|c|}{ Local } & \multirow[t]{2}{*}{ CPR } & \multirow[t]{2}{*}{$\operatorname{aPR}(95 \% \mathrm{Cl})^{\mathrm{a}}$} \\
\hline & $\mathrm{n} / \mathrm{N}$ & $\%$ & $n / N$ & $\%$ & & \\
\hline $6-11$ & $135 / 298$ & 45.3 & $26 / 111$ & 23.4 & 1.93 & $1.93(1.27,2.94)$ \\
\hline $12-17$ & $71 / 194$ & 36.6 & $21 / 106$ & 19.8 & 1.85 & $1.85(1.14,3.01)$ \\
\hline $18-23$ & $38 / 175$ & 21.7 & $13 / 104$ & 12.5 & 1.74 & $1.78(0.95,3.35)$ \\
\hline Total & $244 / 667$ & 36.6 & $60 / 321$ & 18.7 & 1.96 & $1.86(1.40,2.47)$ \\
\hline
\end{tabular}

*Crude and adjusted prevalence ratio (CPR) were estimated by using Poisson regression.

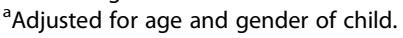

older as $\mathrm{aPR}=1.93$, (95\% CI 1.27 to 2.94$)$ among children aged 6-11 months, aPR $=1.85$, (95\% CI 1.14 to 3.01$)$ among $12-17$ months and $\mathrm{aPR}=1.78$ (95\% CI 0.95 to 3.35$)$ among 18-23 months respectively (Figure 1).

\section{Associated factors}

Table 4 presented the anemia prevalence and potential associated factors in migrant and local children. The 6-11 months and 12-17 months age groups had a higher anemia prevalence compared with the 18-23 months group among the migrant. Anemia was more prevalent among migrant children with breastfeeding at 6 months, didn't consume iron-rich and/or iron fortified foods during previous day. Local children whose mothers were not working for payment and who were from family with more than one child were more likely to be anemic.

Table 5 presented the analysis of association between social-economic status, feeding behaviors and anemia prevalence among migrant and local children respectively by using three adjusted multiple Poisson regression models. In model 1 , in both migrant and local groups, having elder sibling/s were found to be postitively associated with anemia, along with the aPR 1.47 (95\% CI 1.16 to 1.87 ) among migrant children and 2.58 (95\% CI 1.37 to 4.85 ) among local ones. In model 2, continued breastfeeding for children aged 6 months (aPR 1.57, 95\% CI 1.152 to 2.14) were positively and consumption of iron-rich and/or iron fortified foods (aPR 0.68, 95\% CI 0.50 to 0.89) negatively associated with anemia among migrant children. In model 3 , no statistically significant association between MDD, MMF and anemia among both migrant and local children were found.

\section{Discussion}

Based on field survey, we reported the results of a population-based cross-sectional study conducted in Pinghu, an eastern coastal city in China. Comparison of the anemia prevalence and associated factors between migrant children and local children aged 6-23 months had been presented.

Overall anemia prevalence in Pinghu was reported as higher than our assumption, which was based on other researches in China [23-25]. Anemia prevalence among migrant and local children in our study was found to be moderate and mild according to WHO classification [26]. Migrant children had higher anemia prevalence than the local children amongst all age groups. The disparity corresponded to other reports from some big cities such as Shenzhen (9.5\% vs. 7.7\%) [17] and Xiamen (13.1\% vs. 7.3\%) [18]. Overall the disparity of anemia prevalence

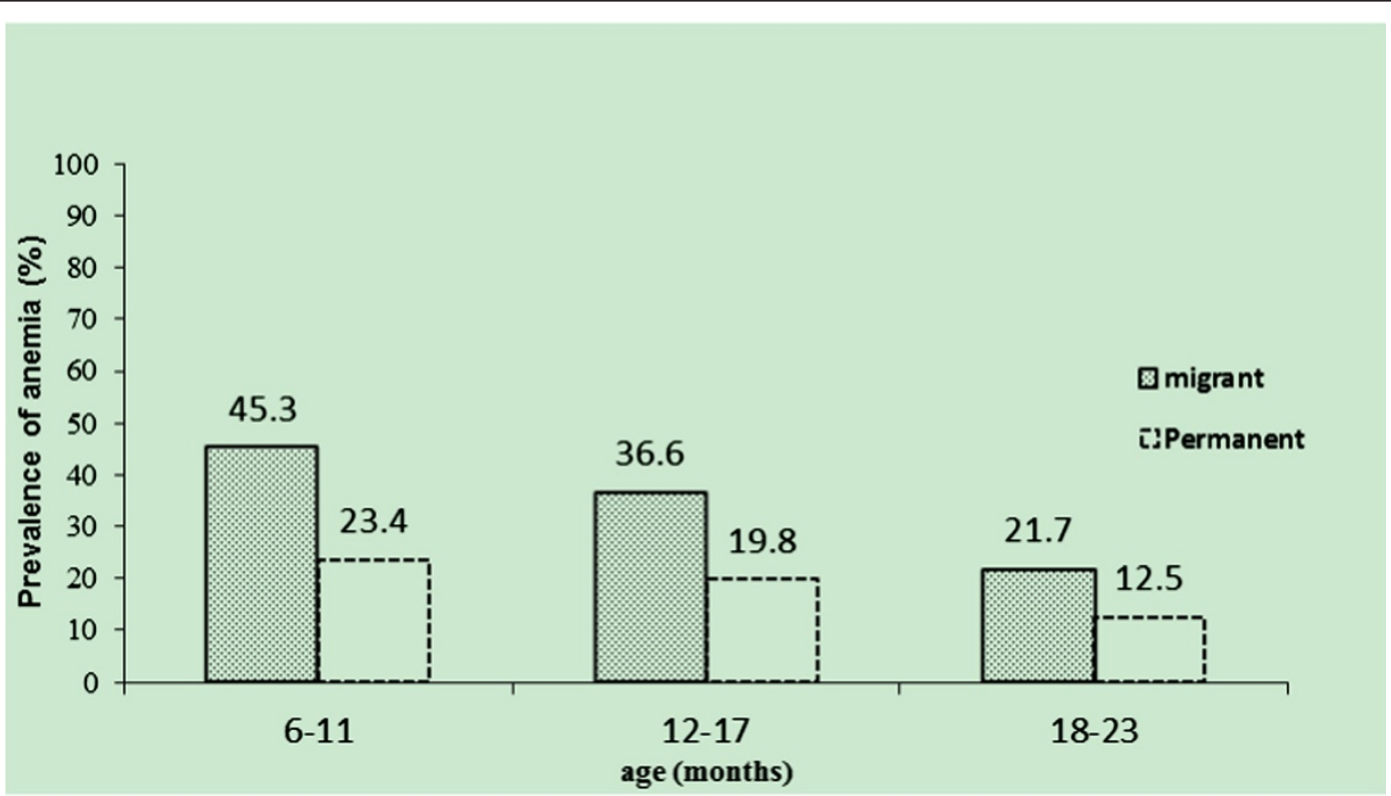

Figure 1 Anemia prevalence among migrant and local children aged 6-23 months. (Cochran's Mantel-Haenszel statistics $(C M H) P<0.001)$. 
Table 4 Prevalence of anemia in migrant and local children of Pinghu China*

\begin{tabular}{|c|c|c|c|c|}
\hline & \multicolumn{2}{|c|}{ Migrant(N = 667) } & \multicolumn{2}{|c|}{ Local $(\mathrm{N}=321)$} \\
\hline & Prevalence of anemia, $\%(\mathrm{n} / \mathrm{N})$ & cPR $(95 \% \mathrm{Cl})$ & Prevalence of anemia, $\%(n / N)$ & CPR $(95 \% \mathrm{Cl})$ \\
\hline \multicolumn{5}{|c|}{ Age of child (months) } \\
\hline $6 \sim$ & $45.3(135 / 298)$ & $2.09(1.46,2.99)$ & $23.4(26 / 111)$ & $1.87(0.96,3.65)$ \\
\hline $12 \sim$ & $36.6(71 / 194)$ & $1.69(1.14,2.50)$ & $19.8(21 / 106)$ & $1.58(0.79,3.17)$ \\
\hline $18 \sim 23$ & $21.7(38 / 175)$ & 1.0 & $12.5(13 / 104)$ & 1.0 \\
\hline \multicolumn{5}{|l|}{ Gender of child } \\
\hline Boy & $36.0(135 / 375)$ & $0.96(0.75,1.24)$ & $17.8(31 / 174)$ & $0.90(0.54,1.50)$ \\
\hline Girl & $37.3(109 / 292)$ & 1.0 & $19.7(29 / 147)$ & 1.0 \\
\hline \multicolumn{5}{|l|}{ Education of mother } \\
\hline Low & $37.1(65 / 175)$ & $0.93(0.60,1.45)$ & $14.3(2 / 14)$ & $1.37(0.32,5.77)$ \\
\hline Medium & $35.8(150 / 419)$ & $0.90(0.61,1.34)$ & $19.5(26 / 133)$ & $1.29(0.31,5.37)$ \\
\hline High & $39.7(29 / 73)$ & 1.0 & $18.4(32 / 174)$ & 1.0 \\
\hline \multicolumn{5}{|l|}{ Occupation of mother } \\
\hline Not work for pay & $40.6(149 / 367)$ & $1.28(0.99,1.66)$ & $27.8(20 / 72)$ & $1.73(1.01,2.96)$ \\
\hline Employed & $31.7(95 / 300)$ & 1.0 & $16.1(40 / 249)$ & 1.0 \\
\hline \multicolumn{5}{|c|}{ Annual family income } \\
\hline Poor & $39.1(156 / 399)$ & $1.16(0.87,1.55)$ & $25.0(19 / 76)$ & $1.38(0.72,2.66)$ \\
\hline Middle & $33.7(64 / 190)$ & $0.91(0.57,1.46)$ & $15.9(24 / 151)$ & $0.88(0.47,1.64)$ \\
\hline Rich & $30.8(24 / 78)$ & 1.0 & $18.1(17 / 94)$ & 1.0 \\
\hline \multicolumn{5}{|l|}{ Caregivers } \\
\hline Parents only & $37.1(138 / 372)$ & $1.03(0.80,1.33)$ & $13.8(4 / 29)$ & $0.72(0.26,1.98)$ \\
\hline Parents \& others & $35.9(106 / 295)$ & 1.0 & $19.2(56 / 292)$ & 1.0 \\
\hline \multicolumn{5}{|l|}{ Elder sibling/s } \\
\hline Yes & $41.1(102 / 248)$ & $1.21(0.94,1.57)$ & $36.0(18 / 50)$ & $2.32(1.34,4.03)$ \\
\hline No & $33.9(142 / 419)$ & 1.0 & $15.5(42 / 271)$ & 1.0 \\
\hline \multicolumn{5}{|c|}{ Timely child health care } \\
\hline Yes & $22.9(8 / 35)$ & $0.61(0.30,1.24)$ & $18.9(14 / 74)$ & $1.02(0.56,1.85)$ \\
\hline No & $37.3(236 / 632)$ & 1.0 & $18.6(46 / 247)$ & 1.0 \\
\hline \multicolumn{5}{|c|}{ Breastfeeding at 6 months } \\
\hline Yes & $40.0(209 / 523)$ & $1.64(1.15,2.35)$ & $21.7(46 / 212)$ & $1.69(0.93,3.07)$ \\
\hline No & $24.3(35 / 144)$ & 1.0 & $12.8(14 / 109)$ & 1.0 \\
\hline \multicolumn{5}{|l|}{ Iron } \\
\hline Yes & $32.3(180 / 557)$ & $0.56(0.42,0.74)$ & $18.9(59 / 312)$ & $1.70(0.24,12.28)$ \\
\hline No & $58.2(64 / 110)$ & 1.0 & $11.1(1 / 9)$ & 1.0 \\
\hline \multicolumn{5}{|l|}{ MDD } \\
\hline Yes & $33.9(163 / 481)$ & $0.78(0.60,1.02)$ & $18.7(53 / 283)$ & $1.02(0.46,2.24)$ \\
\hline No & $43.5(81 / 186)$ & 1.0 & 18.4(7/38) & 1.0 \\
\hline \multicolumn{5}{|l|}{ MMF } \\
\hline Yes & $34.3(168 / 490)$ & $0.80(0.61,1.05)$ & $16.1(45 / 279)$ & $0.45(0.25,0.81)$ \\
\hline No & $42.9(76 / 177)$ & 1.0 & $35.7(15 / 42)$ & 1.0 \\
\hline
\end{tabular}

*Estimated by using Poisson regression, Crude Prevalence Ratios (cPR) and its $95 \% \mathrm{Cl}$ presented.

among migrant and local children in Pinghu (36.6\% vs. $18.7 \%)$ was higher and the disparity was larger than other findings. Moreover, the anemia prevalence among migrant children was similar with the level in rural areas of middle and western China (32\% to 46\%) [23,31-33]. This could partly be explained by the fact that most 
Table 5 Feeding factors associated with anemia in migrant and local children of Pinghu China*

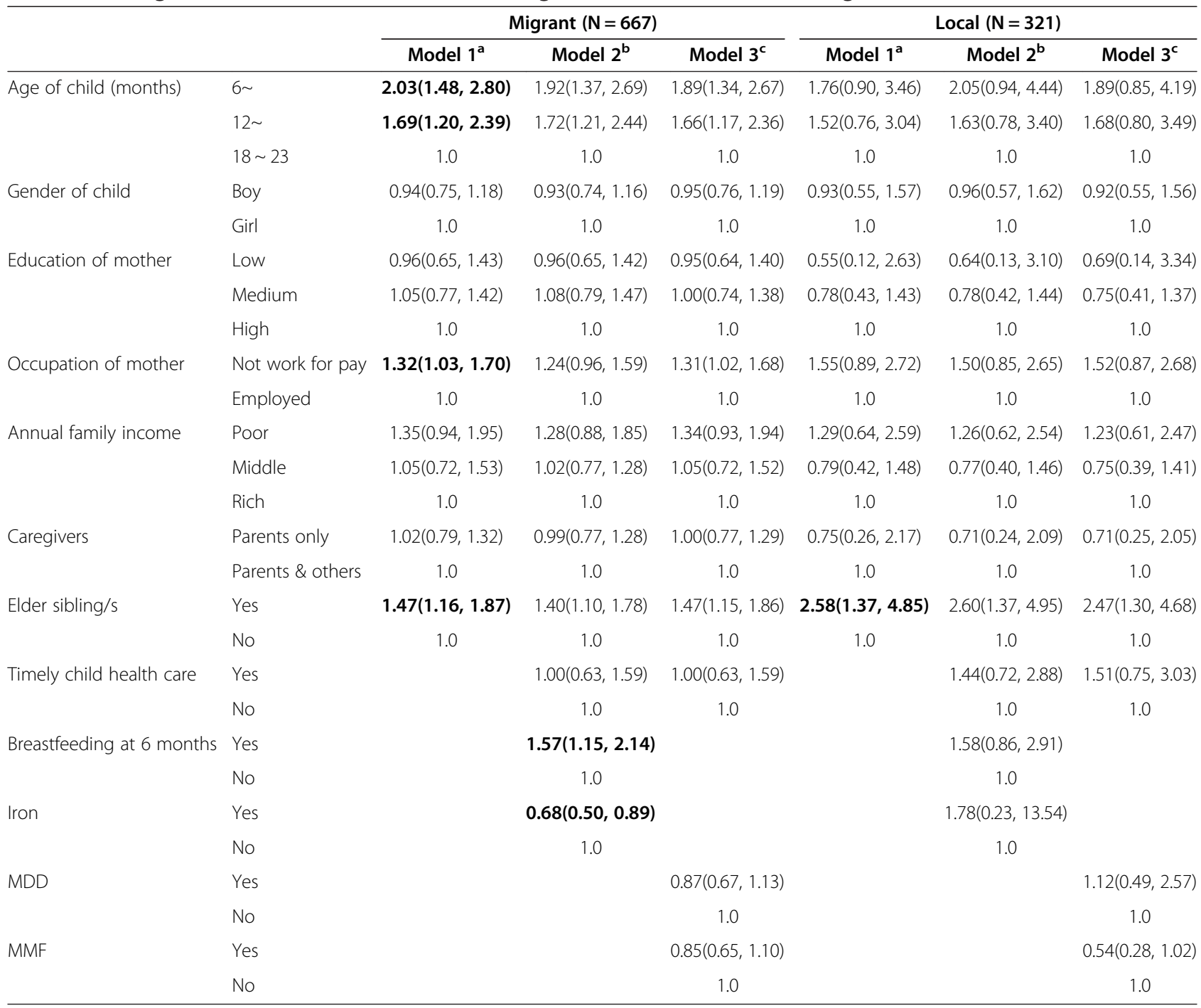

*Estimated by using multiple Poisson regression, adjusted Prevalence Ratios (aPR) and its 95\% Cl presented.

${ }^{a}$ Model 1, the association between age and sex of children; education level and occupation of mother; annual family income, family structure (caregiver/s), number of children (with or without sibling/s) and timely child health care were analyzed.

${ }^{\mathrm{b}}$ Model 2, the association between breastfeeding at 6 months, iron intake and anemia were analyzed, adjusted for age and sex of children; education level and occupation of mother; annual family income, family structure (caregiver/s), number of children (with or without sibling/s) and timely child health care.

${ }^{c}$ Model 3, the association between MDD, MMF and anemia were analyzed, adjusted for age and sex of children; education level and occupation of mother; annual family income, family structure (caregiver/s), number of children (with or without sibling/s) and timely child health care.

migrants in Pinghu were originally from middle and western of China, therefore maintain a similar susceptibility pattern. It also suggests that the process of acculturation of the migrants didn't significantly influence the prevalence of anemia among their children. Another possible explanation could be, as a recent urbanization area, Pinghu was still relatively under-developed areas compared with big cities like Shenzhen and Xiamen. Health services and health of local people lagged behind the industrial development. The development status of Pinghu City, however, was likely to be representative of other urban settlements transformed from traditional rural areas in current China.
Recently the central government of China had launched a nutritional improvement campaign (through delivery of micro-nutrients packages) in western China [34]. But the needs of those children from western part of China who had left for urban life (commonly in coastal or eastern part of the country) had been under covered by public services in their destination of migration. Our findings suggested that anemia among young children in newly-developing areas still deserves higher attention, especially among migrant children at early life. Both migrant and local children had a higher likelihood of anemia in the 6-11 age groups. This corresponded with the findings that children around 
6 months were more vulnerable to anemia [15], especially among migrants, probably due to the limited iron blood reserves at birth.

Environmental factors, especially socio-economic status and feeding practices play leading roles in determining nutritional status in the early years of life. The association between anemia prevalence among young children and social economic status of their families may have multiple pathways [30]. One pathway could be low social economic status associated with antenatal anemia. It then contributes to low birth weight and prematurity, both of which increase the risk of childhood anemia [35]. But socioeconomic status (SES), including mother's education and occupation status, or family income identified as determinants anemia in previous studies [31], did not appear to be significant in our study. No association was observed between children's anemia and maternal schooling in the present study, confirming the findings of Hadlers et al. in Brazil [36]. One possible reason was socioeconomic factors affect health by means of more proximate (intermediate) determinants such as feeding practices which played dominant role to occurrence of childhood anemic other than SES status. In our study, child with sibling/s in both migrant and local group were more likely to be anemic. This associated factor had been previous reported by Yang et al. in China [33,36] which needed further study to explore the reasons.

Accumulating evidences demonstrate that anemia is a common clinical manifestation of micronutrient deficiency, particularly iron, zinc, vitamin $A$, vitamin $B_{12}$, vitamin $B_{2}$, folic acid and magnesium [37-42]. Iron deficiency anemia (IDA) is considered the leading contribution to the global burden of anemia [43]. Introduction of complementary food at early age has prominent effect on iron deficiency correction $[30,44,45]$. These evidences could partly explain the contribution of the influence of food consumption and bioavailability to the IDA. In our study, dietary factors were one of the more significant associated factors to be considered. On one aspect, prolonged exclusively breastfeeding, such as over 6 months old, was reported to be predictor of infant anemia in developing countries [46]. The worsen complementary feeding such as not reaching minimum dietary diversity and minimum meal frequency, lower intake of iron-rich and/or iron-fortified foods especially among migrant children could contribute to low iron blood level among young children which caused higher anemia prevalence among the group. We found the migrant child who was breastfed at aged 6 months and lack of iron supplements was significant associated with anemia prevalence. These two associated factors were not significant in local children. Higher proportion of breastfeeding and lower of complementary diversity hinted there might be a later introduction and lower quality of complementary foods in migrant children. For local children, iron supplement probably not played important role as it to migrant children. Instead, other micronutrient factors, such as Vitamin $B_{2}$ intake [38] probably were more important. All these suggested that breastfeeding and lack of iron supplements were not necessarily increasing the risk of childhood anemia unless over reliance on it for nutrition after aged 6 months.

A few study limitations existed. First, the survey was only based on one city, Pinghu. In order to obtain a clearer picture, future researches should be expanded into other areas, considering the variations across geographic regions. Second, the sample size of local children was defined by the expected anemia prevalence in migrant children in our multiple-indicator study. It might decrease the test effect of the multiple regression analysis. Third, direct epidemiology inference for causality could not be determined due to the cross-sectional design. Bias might also exist, for instance, hemoglobin values measured in capillary samples are higher than in venous, potentially leading to false-negative results [43].

\section{Conclusions}

In conclusion, this study demonstrated anemia prevalence was relatively high among children aged 6-23 months in Pinghu, an eastern coastal city of China. The migrant children were almost twice as likely to be anemic than the local children. Our study also hinted migrant and local children could share same associated factors (with elder siblings) and had their distinct associated factors (continued breastfeeding at aged 6 months and lower intake of iron-rich and/or iron-fortified foods among migrant children but not among local children). Disadvantageous socio-demographic characteristics and inappropriate feeding practices were highly associated with anemia in migrant and local children. Public policies aimed to narrow the nutrition gap between the migrant and local children should target more susceptible groups and through improvement of feeding practices among younger children in those kind of newly-developing areas of China.

\section{Competing interests}

The authors declare that they have no competing interests.

\section{Authors' contributions}

$X Q$ and SFG conceived and XQ, SYH and SFG designed the study. SYH and $\mathrm{XQ}$ developed the field methodology and directed the field work. APP, HJ and JMW helped the data collection and conducted quality control in the field. SYH, HT and HJ analyzed the data. SYH and HT drafted the manuscript. SFG offered valued revision comments. All authors read and approved the final manuscript.

Authors' information

Hui Tan is co-first author.

\section{Acknowledgements}

Authors are grateful to the caregivers and kids participated in the study. This study was supported by grant funding from UNICEF and additional funds from Fudan University. We acknowledge the field team led by Ms. Min Xu and Mr. Yongjie Zhang and logistic supports from Ms. Chunxia Zhao. We 
also thank Professor Naiqing Zhao for his inputs on the statistical analysis and Ms. Liang Guo and Dr. Alison Morgan for English editing.

\section{Author details}

${ }^{1}$ School of Public Health, Fudan University, Shanghai, China. ${ }^{2}$ Global Health Institute, Fudan University, Shanghai, China. ${ }^{3}$ Key Laboratory of Public Health Safety, Ministry of Education, Shanghai, China. ${ }^{4}$ Shanghai Maternal and Child Health Center, Shanghai, China. ${ }^{5}$ Pinghu Institute of Maternal and Child Health, Zhejiang Province, China. ${ }^{6}$ United Nations Children's Fund China Country Office, Beijing, China.

Received: 13 October 2013 Accepted: 8 June 2014

Published: 14 June 2014

\section{References}

1. Seeborg MC, Jin Z, Zhu Y: The new rural-urban labor mobility in China: causes and implications. J Socio-Econ 2000, 29(1):39-56.

2. Wakabayashi K: Migration from rural to urban areas in China. Dev Econ 1990, 28(4):503-523.

3. Shen JJ: Changing patterns and determinants of interprovincial migration in China. Popul Space Place 2012, 18(3):384-402.

4. National Bureau of Statistics of China: China Statistic Year Book. Beijing: China Statistics Press; 2012.

5. Woetzel J, Mendonca L, Devan J, Negri S, Hu Y, Jordan L, Li X, Maasry A, Tsen G, Yu F: Preparing for China's urban billion. Shanghai: Mckinsey Global Institute; 2009. Available at: http://www.mckinsey.com/insights/ urbanization/preparing_for_urban_billion_in_china. [Accessed 30 Septermber 2012]

6. Yusuf S, Saich T: China urbanizes: consequences, strategies, and policies. Washington DC: World Bank Publications; 2008.

7. Chen W, Liu J: Family patterns of the floating population in Beijing. Popul J 2012, 196(06):3-8.

8. Duan $C R$, Yang K: Study on the latest situation of floating children in China: a study based on sampled survey among $1 \%$ national-wide population in 2005. Popul J 2005, 2008(6):23-31.

9. Gong P, Liang S, Carlton EJ, Jiang Q, Wu J, Wang L, Remais JV: Urbanisation and health in China. Lancet 2012, 379(9818):843-852.

10. Salant T, Lauderdale DS: Measuring culture: a critical review of acculturation and health in Asian immigrant populations. Soc Sci Med 2003, 57(1):71-90.

11. Frisbie WP, Cho Y, Hummer RA: Immigration and the health of Asian and Pacific Islander adults in the United States. Am J Epidemiol 2001, 153(4):372-380.

12. Bao SM, Bodvarsson OB, Hou JW, Zhao YH: The regulation of migration in a transition economy: China's Hukou system. Contemp Econ Policy 2011, 29(4):564-579

13. Kuang $L$, Liu L: Discrimination against rural-to-urban migrants: the role of the hukou system in China. Plos One 2012, 7(11):e46932. doi:10.1371/ journal.pone.0046932.

14. Yan S, Chen X, Duan J, Liu G: State and demand of health care service among floating children under 5 year old in Beijing. Chinese J Child Health Care 2008, 16(5):542-543.

15. Black RE, Victora CG, Walker SP, Bhutta ZA, Christian P, Onis MD, Ezzati M, Grantham-Mcgregor S, Katz J, Martorel RM, Uauy R: Maternal and child undernutrition and overweight in low-income and middle-income countries. Lancet 2013, 382(9890):427-451. http://dx.doi.org/10.1016/ S0140-6736(13)60937-X

16. Lozoff B, Jimenez E, Smith JB: Double burden of iron deficiency in infancy and low socioeconomic status: a longtudinal analysis of cognitive test scores to age 19 years. Arch Pediatr Adolesc Med 2006, 160(11):1108-1113.

17. Gao W, Wang Q, Xi YJ: Survey on anaemia prevalence among 4127 children aged 0 to 3 years in Shenzhen. Matern Child Health China 2011, 29:4586.

18. Ai XL: Analysis of anaemia among 9481 children aged 6 to 59 months in Canghai District of Xiamen. Chinese J Child Health Care 2012, 20(12):1146-1148.

19. Ding WQ, Feng SF: Analysis of the state of health care service among floating children and resident children under 5 years old in Yinchuan. Modern Preventive Medicine 2011, 38(20):4130-4132.

20. Shi $X$, Lin $X Q$, Liang $H N$, Tan HW, Long $X$ : Anaemia among preschool children and its relationship with parents' KAP in Nanning. Chinese J Child Health Care 2008, 29(9):839-840.
21. WHO: Maternal, newborn and child health household survey guidelines (Draft). Switzerland: Geneva: World Health Organization; 2009.

22. Chang S, He W, Jia F, Chen C: Analysis on the changes of nutritional status in China: anemia status of children under 5 in China. J Hygiene Res 2007, 36(2):210-212.

23. Cui Y, Qu Q, Yang L, Wu Q: Analysis on anemia status of children under 3 years old in western China. Chinese J Public Health 2008, 24(9):1052-1053.

24. Chang SY, He W, Chen CM: The Growth characteristics of children under 5 in the past 15 years: changing pattern of growth and development of children under age 5. J Hygiene Res 2006, 35(6):768-771.

25. Ba L: Anemia investigation of migrant children under 5 in Chaoyang district. Chinese J Child Health Care 2011, 19(4):357-359.

26. World Health Organization (WHO): Haemoglobin concentrations for the diagnosis of anaemia and assessment of severity. In Vitamin and Mineral Nutrition Information System. Geneva: World Health Organization; 2011. WHO/NMH/11.1) Available at: http://www.who.int/vmnis/indicators/ haemoglobin.pdf, [Accessed 25 October 2011]

27. World Health Organization (WHO): Indicators for assessing infant and young child feeding practices: Part 1 Definitions. World Health Organization; 2008. Available at: http://whqlibdoc.who.int/publications/ 2010/9789241599290 eng.pdf [Accessed 25 October 2011]).

28. Black RE, Allen LH, Bhutta ZA, Caulfield LE, de Onis M, Ezzati M, Mathers $C$, Rivera J: Maternal and child undernutrition: global and regional exposures and health consequences. Lancet 2008, 371(9608):243-260.

29. Pasricha SR, Black J, Muthayya S, Shet A, Bhat V, Nagaraj S, Prashanth NS, Sudarshan H, Biggs BA, Shet AS: Determinants of anemia among young children in rural India. Pediatrics 2010, 126(1):e140-e149.

30. Os O, Rio MM: Determinant factors of anemia in children. J Pediatr (Rio J) 2002, 78(4):269-278.

31. Zeng $L$, Yan H, Chen Z, Dang S, Xie H: Analysis on the prevalence of anemia among children under 3 -year-old in 5 provinces in Western China. Chin J Epidemiol 2004, 25(3):47-50.

32. Zhang A, Zhang J, Wang Y: Study on anemia of $6 \sim 35$ months old children in 46 counties of midwestern China. Chinese J Child Health Care 2009, 17(3):260-261.

33. Yang WF, Li X, Li Y, Zhang SP, Liu SP, Wang X, Li WM: Anemia, malnutrition and their correlations with socio-demographic characteristics and feeding practices among infants aged $0-18$ months in rural areas of Shaanxi province in northwestern China: a cross-sectional study. BMC Public Health 2012, 12:1127.

34. Ministry Of Health, P.R.China: Report on nutrition status improvement of children aged 0 to 6 in China (2012). China: Beijing: 2012. Available at: http://www.moh.gov.cn/cmsresources/mohjcg/cmsisdocument/doc15032.doc, [Accessed 25 October 2013]

35. Wharton BA: Iron deficiency in children: detection and prevention. $\mathrm{Br} J$ Haematol 1999, 106(2):270-280.

36. Ma Y, Bi Y, Yan H, Deng L, Liang W, Wang B, Zhang X: The application of decision tree in the research of anemia among rural children under 3-year-old. Zhonghua Yu Fang Yi Xue Za Zhi 2009, 43(5):434-437.

37. Hadler MC, Juliano Y, Sigulem DM: Anemia in infancy: etiology and prevalence. J Pediatr (Rio J) 2002, 78(4):321-326.

38. Shi Z, Zhen S, Wittert GA, Yuan B, Zuo H, Taylor AW: Inadequate riboflavin intake and anemia risk in a Chinese population: five-year follow up of the Jiangsu nutrition study. PLoS One 2014, 9(2):e88862. doi:10.1371/ journal.pone.0088862.

39. Lynch S, Stoltzfus R, Rawat R: Critical review of strategies to prevent and control iron deficiency in children. Food Nutr Bull 2007, 28(4 Suppl):S610-S620.

40. Calis JC, Phiri KS, Faragher EB, Brabin BJ, Bates I, Cuevas LE, Haan RJ, Phiri Al, Malange P, Khoka M, Hulshof PJM, Lieshout L, Beld MGHM, Teo YY, Rockett KA, Richardson A, Kwiatkowski DP, Molyneux M, Hensbroek MB: Severe anemia in Malawian children. N Engl J Med 2008, 358(9):888-899.

41. Zhan Y, Chen R, Zheng W, Guo C, Lu L, Ji X, Chi Z, Yu J: Association between serum magnesium and anemia: China health and nutrition survey. Biol Trace Elem Res 2014, doi:10.1007/s12011-014-9967-x.

42. Shi Z, Hu X, He K, Yuan B, Garg M: Joint association of magnesium and iron intake with anemia among Chinese adults. Nutrition 2008, 24(10):977-84.

43. Benoist B, McLean E, Egli I, Cogswell O: Worldwide prevalence of anaemia 1993-2005: WHO Global Database on Anaemia. Switzerland: Geneva: World Health Orgranization; 2008. Available at: http://www.who.int/vmnis/ publications/anaemia_prevalence/en/, [Accessed 1 Sep, 2012] 
44. Leung AK, Chan KW: Iron deficiency anemia. Adv Pediatr 2001, 48:385-408.

45. WHO, UNICEF, UNU: Iron deficiency anaemia: assessment, prevention and control, a guide for programme managers. Geneva: World Health Organization; 2001. Available at: http://www.who.int/nutrition/publications/ micronutrients/anaemia_iron_deficiency/WHO_NHD_01.3/en/index.htm. [Accessed 1 Sep, 2012]

46. Meinzen-Derr JK, Guerrero ML, Altaye M, Ortega-Gallegos H, Ruiz-Palacios GM, Morrow AL: Risk of infant anemia is associated with exclusive breast-feeding and maternal anemia in a Mexican cohort. J Nutr 2006, 136(2):452-458.

doi:10.1186/1471-2458-14-601

Cite this article as: Hu et al:: Disparity of anemia prevalence and associated factors among rural to urban migrant and the local children under two years old: a population based cross-sectional study in Pinghu, China. BMC Public Health 2014 14:601.

\section{Submit your next manuscript to BioMed Central and take full advantage of:}

- Convenient online submission

- Thorough peer review

- No space constraints or color figure charges

- Immediate publication on acceptance

- Inclusion in PubMed, CAS, Scopus and Google Scholar

- Research which is freely available for redistribution 